\title{
ANALYSIS OF GOLD MICRO-BEAMS WITH MODIFIED STRAIN GRADIENT THEORY
}

\author{
Hüsnü DAL * \\ Department of Mechanical Engineering, Faculty of Engineering, Middle East Technical University, \\ TR06800, Ankara, Turkey
}

\begin{abstract}
Micro-beams are building blocks for many micro- and nano-electro-mechanical systems (MEMS/NEMS) and cannot accurately be modeled by classical continuum theories due to significant size effects at the length scales associated with these structures. Size effects can be taken into account by the so-called higher-order continuum theories. In this study, Euler-Bernoulli microbeams are analyzed with the Modified Strain Gradient Theory (MSGT), which extends the classical local continuum theories of grade one with the introduction of three additional length scale parameters. In this contribution, finite element implementation is briefly demonstrated by using Galerkin discretization techniques for Euler-Bernoulli beams. The size effect for gold-micro beams is demonstrated and the length scale parameters of gold micro-beams for MSGT are identified from the existing experimental data from literature for the first time. As a novel aspect, significant size effect is demonstrated for the length-scales associated with the state of the art gold micro-beam structures developed for MEMS and NEMS applications, which reveals the necessity of the use of higher-order theories at these length scales.
\end{abstract}

Keywords: Gold micro-beams, Modified strain gradient theory, Higher-order elasticity, Size effect, MEMS / NEMS devices

\section{INTRODUCTION}

Micro- and nano-electro-mechanical systems (MEMS/NEMS) are integral parts of the modern world and their application areas are steadily growing thanks to their small sizes, low weights, ease of implementation in integrated circuits, low energy consumption, and manufacturing costs, earning them vast utilization areas from automotive to defense industry, from biomedical engineering to consumer electronics, and from optics to communication systems. Several products in these areas can be counted as metering devices, sensors, computer hardware, micro-power terminals, RF-MEMS and relevant equipment. MEMS devices can be made of metallic, polymeric, silicon-based or functionally graded materials. It must be noted that the most common deformation modes analyzed and modeled in literature are bending, vibration, and buckling, yet depending on specific applications. The most common types of micro-beams encountered in MEMS design are double-cantilever, cantilever, and simply supported beams where the classical Euler Bernoulli Beam theory is the most suitable mathematical model for the analysis.

Recently, the feature size of the state of the art NEMS/MEMS devices has approached the nanometer scale [2]. As these deformations are to remain mostly in the elastic regime, the elastic material parameters and the mechanical behavior of micro and nano-sized materials should be assessed accurately at this length scale. Gold possesses excellent mechanical and electrical properties, such as high electrical conductivity, chemical inertness, resistance to surface wear, relatively lower residual stress after manufacturing due to thermo-mechanical loads, and relatively lower thermal fluctuation. These properties make gold popular for MEMS/NEMS applications [1-4]. Hence, the mechanical behavior of gold under bending dominated loading conditions at micron scale will be targeted in this study. 
The fact that most engineering materials exhibit size dependent mechanical response is a well known phenomenon for a long time [5-7]. This phenomenon is known as size effect or scale effect and it affects the material properties such as strength, elastic response, hardness, and isotropy. Size effect in gold micro-structures was experimentally shown under tensile [8], compressive [9], bending [10] tests respectively. The size effect in engineering materials dates back to early work of Voigt [11]. The early works were oriented towards understanding the physical phenomena rather than defining mathematical and continuum models. Numerous contributions in explaining size effect in micro-structures have been made in 1960's. Within this context Couple Stress Theory (CST) was introduced by Mindlin and Tiersten [12] as an extension to Voigt's work where surface loads are in fact both force and moment vectors. Various continuum approaches were presented in e.g. Grioli [13], Aero and Kuvshinskii [14], Eringen [15], and Nowacki [16]. General higher-order theories with improved accuracy for finding solutions to singularity problems in classical elasticity theory, e.g. concentrated loads, regions around crack tips, negative slope regime in stress-strain curve of strain softening were also developed. Works of Toupin [17], Mindlin [18, 19], Mindlin and Eshel [20], and Koiter [21] and can be counted as the originators of the higher-order elasticity theories. These are generally categorized as couple-stress theories (CST) and strain-gradient theories (SGT). The primary difference between CST and SGT was the inclusion of symmetric part of second-order deformation gradient in SGT which leads to three additional length-scale parameters. Hence, SGT includes five length-scale parameters whereas CST includes only two. In this way, CST can be considered as a mathematically more simple form of SGT. Fleck and Hutchinson used the previous concept of strain gradient and extended it in an improved SGT framework of higher order theories, which is also implemented in this study [23]. This approach was originally developed for plasticity problems [6, 22]. Lam et al. [25] decreased this number from five to three in SGT using new equilibrium conditions. Yang et al. [24] introduced a new equilibrium equation in CST, which decreased the number of independent length scale parameters from two to one. The studies by Lam et al. [25] and Yang et al. [24] led the way to what is currently known as modified straingradient theory (MSGT) and modified couple-stress theory (MCST) in the literature, where the number of length scale parameters are further reduced to three and one, respectively.

MSGT proposed by Lam et al. [25], is proven to be an accurate, consistent, and mathematically complete model especially when applied to beams as concluded by various studies in the literature. This enabled the use of MSGT in the analysis of micro-structure and MEMS devices. Kong et al. [26] used this extended theory to solve bending and vibration problems for Euler-Bernoulli beams. Wang et al. [27] developed micro-scale Timoshenko and Euler-Bernoulli micro-beam models, which recover the classical Timoshenko and Euler-Bernoulli beam theories for vanishing length scale parameters. They solved bending and free vibration problems for a simply supported Timoshenko beam with this model. Kahrobaiyan et al. [28, 29] analyzed non-linear Euler-Bernoulli micro-beams and developed accurate size-dependent strain-gradient beam finite elements and compared them with readily available experimental data. Akgöz and Civalek studied bending behavior of an axially loaded micro-beam [30] as well as deriving analytical formulations for various boundary conditions for Euler-Bernoulli microbeams [31], as well as developing beam and plate models which captures the microstructural and shear deformation effects without shear correction factors [32, 33, 34]. Zhao et al. [35] derived a nonlinear governing equation including Hamilton's principle, also considering residual stresses that exist as a result of micromachining. Ashgari et al. [36] focused on nonlinear Timoshenko beam model for free vibration and static problems using hinged-hinged boundary condition. Vatankhah et al. [37] focused on nonlinear vibrations caused by immovable supports and they considered the nonlinear phenomena that happen in the large amplitude deflections, which is due to the induced mid-plane stretching during transverse deflections. Zhang et al. [38] focused on development of a new Timoshenko beam finite element that can be used with SGT that satisfies $\mathrm{C}_{0}$ continuity and $\mathrm{C}_{1}$ weak continuity, and modeled bending, vibration, buckling type deformation modes. Rafaeifard et al. [39] developed a SGT-based yield criterion that is in satisfactory compliance with experimental findings. Taati et al. [40] pursued thermo-elasticity extension of the theory where SGT is coupled with non-Fourier heat conduction and applied it to a thermo-elastic problem of a simply supported micro-beam. Unlike the statically 
determinate beam formulations proposed in the literature, Triantafyllou and Giannakopoulos [41] argued that MEMS designers need statically indeterminate beam formulations and proposed a Timoshenko beam formulation that can be used in general statically indeterminate problems. Ghayesh et al. [42] studied nonlinear forced vibrations of micro-beams and obtained frequency-response curves for a micro-beam. Ansari et al. [43] analyzed free vibration behavior of functionally graded micro-beams based on the strain gradient Timoshenko beam theory as well as coming up with a parametric study covering the influences of various parameters on the natural frequencies of these micro-beams. Liebold and Müler [44] implemented various higher-order theories including MSGT into finite element method and identified material parameters for epoxy and SU-8 polymer under bending. Papargyri-Beskou et al. [45] used a simple theory of gradient elasticity with surface energy and derived a model of beam bending and buckling. Shojaeian et al. [46] modelled clamped-guided nanobeams under axial loads including the effect of nonlinear electrostatic and van der Waals forces for analysis of electromechanical buckling in NEMS structures.

Despite the well-known fact for the mechanics community that considerable size effect occurs in gold, it has not attracted significant attention in the MEMS-NEMS community so far. This study aims to identify the length scale parameters based on the existing experimental data published in literature for the modified strain gradient theory and assess the order of error introduced by using the classical theory for micro-beams, where to the author's best knowledge, has not been done so far. To this end, the Modified Strain Gradient Theory (MSGT) has been applied to Euler-Bernoulli micro-beams. Within this context, the modified strain gradient theory and the finite element formulation based on Galerkin-type weak formulation is briefly discussed. The finite element method is chosen for two reasons: Firstly, it is the most common numerical analysis tool in engineering practice. Secondly, analytical solutions can only be utilized for well defined geometry and boundary conditions. Finite element method can treat arbitrary beam geometries and boundary conditions in a strainghtforward manner.

The paper is organized as follows: In Section 2, the MSGT is introduced and incorporation into the Euler-Bernoulli beam theory is explained. With the help of the internal energy expression for purely bending deformations, Galerkin type weak form is derived as the first variation of the total potential energy. Finally, the discrete counterpart of the weak form for the finite element method is obtained by incorporating the interpolation (shape) functions leading to the element stiffness matrix. Numerical analysis of double cantilever beams for various geometries are carried out and the length scale parameters of MSGT are identified for gold micro-beams in Section 3. Critical length scale parameters, which necessitate the use of higher-order theories, are identified from existing experimental data from the literature [47]. The results of the paper are discussed in Section 4.

\section{THEORY AND NUMERICAL IMPLEMENTATION}

\subsection{Theoretical Background for Modified Strain Gradient Theory}

The starting point of gradient theories is the introduction of the free energy density function $\Psi$, which, is not only a function of strains (first-order displacement gradients), but also a function of first and second gradient of strains

$$
\Psi=f\left(\varepsilon, \varepsilon^{1}, \varepsilon^{2}\right)
$$

unlike the classical local theories of grade one [48]. Herein, the strain measures

$$
\boldsymbol{\varepsilon}=\frac{1}{2}\left(\nabla \boldsymbol{u}+\nabla^{T} \boldsymbol{u}\right), \boldsymbol{\varepsilon}^{\mathbf{1}}=\nabla \nabla \boldsymbol{u}, \boldsymbol{\varepsilon}^{\mathbf{2}}=\nabla \nabla \nabla \boldsymbol{u}
$$

denote the first, second and third order strain tensors, respectively, where $\boldsymbol{u}$ is the displacement field. This formulation yields eighteen coefficients, two of which are classical Lamé constants $(\lambda$ and $\mu$ ). The 
above given ansatz (1) lead to a mathematically complete and elegant theory requiring 16 additional elastic constants parameters. This causes certain difficulties in the analytical investigations and the parameter identification. The reduced form of the free energy density

$$
\Psi=f\left(\varepsilon_{i j}, \eta_{i j k}\right)
$$

reduces the number of elastic constants to five. Herein,

$$
\varepsilon_{i j}=\frac{1}{2}\left(u_{i, j}+u_{j, i}\right) \quad \text { and } \quad \eta_{i j k}=\partial_{i j} u_{k}
$$

are the conventional strain tensor and the second order displacement gradient tensor in indicial notation, respectively.

Lam et al. [25] expressed the second order displacement gradient tensor

$$
\eta_{i j k}=\eta_{i j k}^{S}+\eta_{i j k}^{A}=\eta_{i j k}^{S(0)}+\eta_{i j k}^{S(1)}+\eta_{i j k}^{A}
$$

i.e. the summation of its symmetric part $\left(\eta_{i j k}^{S}\right)$ and anti-symmetric part $\left(\eta_{i j k}^{A}\right)$ or equivalently, as the summation of the trace part of symmetric part $\left(\eta_{i j k}^{S(0)}\right)$, deviatoric part of the symmetric part $\left(\eta_{i j k}^{S(1)}\right)$, and anti-symmetric part. By neglecting the contributions of the antisymmetric part and the trace part of the second order displacement gradient tensor, the internal strain energy $W^{\text {int }}$ for a linear elastic beam based on MSGT can be expressed in the form

$$
W^{i n t}=\frac{1}{2} \int\left(\sigma_{i j} \varepsilon_{i j}+p_{i} \gamma_{i}+\tau_{i j k}^{S(1)} \eta_{i j k}^{S(1)}+m_{i j}^{S} \chi_{i j}^{S}\right) d V
$$

where the additional deformation measures are defined as

$$
\begin{aligned}
& \gamma_{i}=\varepsilon_{m m, i}=\partial_{i} \varepsilon_{m m}, \\
& \eta_{i j k}^{S(1)}=\frac{1}{3}\left(\partial_{i} \varepsilon_{j k}+\partial_{j} \varepsilon_{k i}+\partial_{k} \varepsilon_{i j}\right)-\frac{1}{15}\left[\delta_{i j}\left(\partial_{k} \varepsilon_{m m}+2 \partial_{m} \varepsilon_{m j}\right)\right. \\
& \left.-\delta_{j k}\left(\partial_{i} \varepsilon_{m m}+2 \partial_{m} \varepsilon_{m i}\right)-\delta_{k i}\left(\partial_{j} \varepsilon_{m m}+2 \partial_{m} \varepsilon_{m j}\right)\right], \text { and } \\
& \chi_{i j}^{S}=\frac{1}{2}\left(e_{i p q} \partial_{p} \varepsilon_{q j}+e_{j p q} \partial_{p} \varepsilon_{q i}\right) .
\end{aligned}
$$

Therein, $\gamma_{i}$ is the dilatation strain gradient vector, $\eta_{i j k}^{S(1)}$ is the deviatoric stretch gradient tensor, and $\chi_{i j}^{S}$ is the symmetric rotational gradient tensor, $\delta_{i j}$ is the Kronecker delta and

$$
e_{i j k}=\left\{\begin{array}{c}
+1 \text { : even permutation }\left(e_{123}, e_{231}, e_{312}\right) \\
-1: \text { odd permutation }\left(e_{132}, e_{213}, e_{321}\right) \\
0: \text { double indexing }\left(e_{112}, e_{121}, \ldots \text { etc. }\right)
\end{array}\right.
$$

is the third order Ricci permutation tensor. The corresponding stress measures, which are the work conjugates of deformation measures, are

$$
\sigma_{i j}=\lambda \delta_{i j} \varepsilon_{m m}+2 \mu \varepsilon_{i j}
$$


Dal / Anadolu Univ. J. of Sci. and Technology A-Appl. Sci. and Eng. 18 (3) - 2017

$$
\begin{gathered}
p_{i}=2 \mu l_{0}{ }^{2} \gamma_{i}, \\
\tau_{i j k}{ }^{S(1)}=2 \mu l_{1}{ }^{2} \eta_{i j k}^{S(1)}, \\
m_{i j}^{S}=2 \mu l_{2}{ }^{2} \chi_{i j}^{S} .
\end{gathered}
$$

Therein, $\sigma_{i j}$ is the Cauchy stress tensor, $p_{i}$ is the pressure gradient, $\tau_{i j k}{ }^{(1)}$ is the double stress tensor, $m_{i j}^{S}$ is the couple stress tensor (symmetric part), and $l_{0}, l_{1}, l_{2}$ are the length scale parameters that are of major concern in this article. Here, the Lamé constants $(\lambda$ and $\mu)$ are expressed as

$$
\lambda=E v /(1+v)(1-2 v), \quad \mu=E / 2(1+v)
$$

where $E$ is the elastic modulus, $v$ is the Poisson's ratio.

\subsection{Euler-Bernoulli Beam}

An Euler-Bernoulli beam under bending is depicted in Figure 1. The basic kinematic assumptions for the deformations are

$$
u=-z \frac{d w(x)}{d x} \quad \text { and } \quad w=w(x)
$$

respectively. Therein $u$ and $w$ corresponds to displacements in $x$ and $z$ directions respectively, $\theta$ is the rotation angle of the centroidal axis of the beam. Plane sections are assumed to remain plane in EulerBernoulli beam theory leading to

$$
\theta=\frac{d w(x)}{d x}
$$

In $y$ direction, plane stress or plane strain conditions can be made according to the width/thickness ratio. For thin films, plane strain conditions will be assumed (as $b / t \geq 5$ for the beams considered in this article as given in Tables 1 and 2 and Figure 3) leading to

$$
\varepsilon_{y y}=\varepsilon_{y x}=\varepsilon_{y z}=0 .
$$

Substituting equation (11) into the conventional strain tensor equation (4), the in-plane strain components are obtained

$$
\varepsilon_{x x}=-z \frac{d^{2} w(x)}{d x^{2}}, \quad \varepsilon_{x z}=\frac{1}{2}\left(\frac{d u}{d z}+\frac{d w}{d x}\right)=\frac{1}{2}\left(-\frac{d w}{d x}+\frac{d w}{d x}\right)=0, \quad \varepsilon_{z z}=\frac{d w}{d z}=0 .
$$

(a)
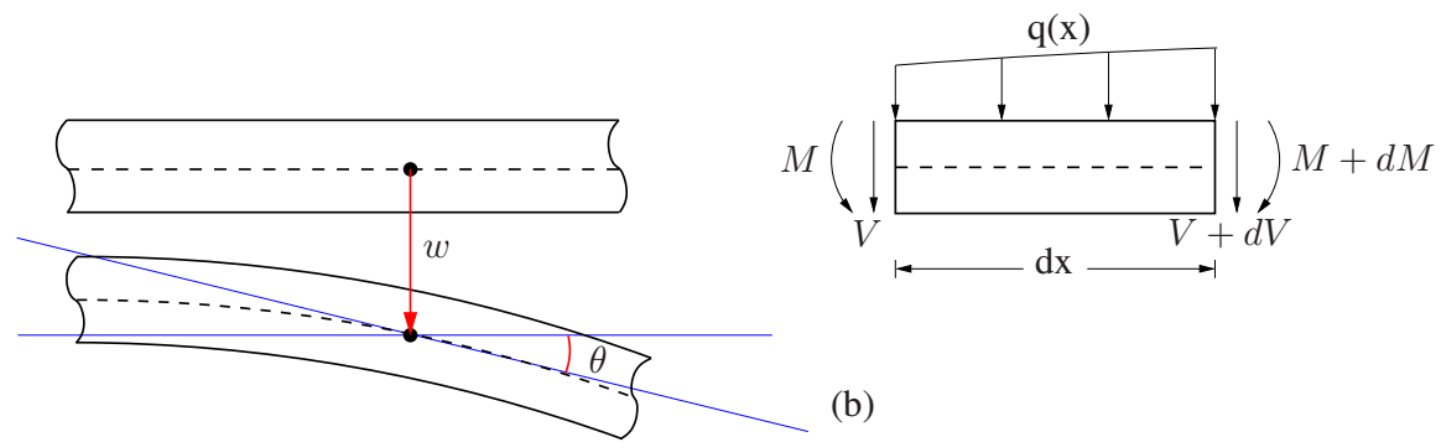

(b)

Figure 1. (a) Coordinate frame and kinematic parameters, (b) forces acting on a differential Euler-Bernoulli beam under bending. 
Then, by substituting equation (9) into equation (14), the stress components are obtained

$$
\begin{gathered}
\sigma_{x x}=\frac{E(1-v)}{(1-2 v)(1+v)}\left(-z \frac{d^{2} w(x)}{d x^{2}}\right), \\
\sigma_{y y}=\sigma_{z z}=\frac{E v}{(1-2 v)(1+v)}\left(-z \frac{d^{2} w(x)}{d x^{2}}\right), \\
\sigma_{x y}=\sigma_{y z}=\sigma_{x z}=0 .
\end{gathered}
$$

The following expression can then be derived for the total potential energy $\Pi=W^{\text {int }}-W^{\text {ext }}$, using equations (6-15)

$$
\Pi=\frac{1}{2} \int_{0}^{L}\left[\left(E^{\prime} I+2 \mu A l_{o}^{2}+\mu A \frac{8}{15} l_{1}^{2}+\mu A l_{2}^{2}\right)\left(\frac{d^{2} w}{d x^{2}}\right)^{2}+\mu I\left(2 l_{o}^{2}+\frac{4}{5} l_{1}^{2}\right)\left(\frac{d^{3} w}{d x^{3}}\right)^{2}\right] d x-W^{e x t},
$$

where $E^{\prime}$ is defined as

$$
E^{\prime}=\frac{E(1-v)}{(1-2 v)(1+v)}
$$

and $I$ is the area moment of inertia, and $A$ is the cross-sectional area of the beam [31].

It must be noted that various authors take different values for $E^{\prime}$ with an additional simplification of disregarding Poisson's ratio $v$ in the derivation of equation (17). Kahrobaiyan et al. [28] take $E^{\prime}=E$ for general case and Zhao et al. [35] take it to be $E^{\prime}=E /\left(1-v^{2}\right)$ for plane strain case. There are also different opinions of the requirement of Poisson's ratio in the relevant formulation [49,50]. The results in this article will be obtained by using equation (17) based on plane strain assumption, but a comparison of length scale parameters based on plane stress assumption is also provided in Section 3.

$W^{\text {ext }}$ is the external work due to applied surface and body forces and is given as:

$$
W^{\text {ext }}=\int_{0}^{L} q(x) w d x+\left.[V(x) w]\right|_{0} ^{L}+\left.\left[M(x) w^{\prime}\right]\right|_{0} ^{L}+\left.\left[Q(x) w^{\prime \prime}\right]\right|_{0} ^{L}
$$

Taking the first variation of (15) and incorporation of

$$
\begin{gathered}
C_{1}=E^{\prime} I+\mu A\left(2 l_{o}^{2}+\frac{8}{15} l_{1}^{2}+l_{2}^{2}\right), \text { and } \\
C_{2}=\mu I\left(2 l_{o}^{2}+\frac{4}{5} l_{1}^{2}\right),
\end{gathered}
$$

leads to

$$
\begin{gathered}
\delta \Pi=\int_{0}^{L}\left[C_{1} w^{(i v)}-C_{2} w^{(v i)}\right] \delta w d x+\left.\left[-C_{1} w^{\prime \prime \prime}+C_{2} w^{(v)}\right] \delta w\right|_{0} ^{L} \\
+\left.\left[C_{1} w^{\prime \prime}-C_{2} w^{(i v)}\right] \delta w^{\prime}\right|_{0} ^{L}+\left.C_{2} w^{\prime \prime \prime} \delta w^{\prime \prime}\right|_{0} ^{L}-\delta w^{e x t}=0 .
\end{gathered}
$$

For the variation $\delta w$, the corresponding external conjugate forces are the shear forces $q(x)$ and $V(x)$, whereas the conjugate forces for the variation of rotation $\delta w^{\prime}$ is the bending moment $M(x)$. The work conjugate of the variational curvature term $\delta w^{\prime \prime}$ is the higher-order load $Q(x)$. Within this context, the first variation of the external $\delta W^{\text {ext }}$ can be described as: 


$$
\delta W^{\text {ext }}=\int_{0}^{L} q(x) \delta w d x+\left.[V(x) \delta w]\right|_{0} ^{L}+\left.\left[M(x) \delta w^{\prime}\right]\right|_{0} ^{L}+\left.\left[Q(x) \delta w^{\prime \prime}\right]\right|_{0} ^{L}
$$

Incorporation of equation (21) into equation (20) results in

$$
\begin{aligned}
\delta \Pi & =\int_{0}^{L}\left[C_{1} w^{(i v)}-C_{2} w^{(v i)}-q(x)\right] \delta w d x+\left.\left[-C_{1} w^{\prime \prime \prime}+C_{2} w^{(v)}-V(x)\right] \delta w\right|_{0} ^{L} \\
& +\left.\left[C_{1} w^{\prime \prime}-C_{2} w^{(i v)}-M(x)\right] \delta w^{\prime}\right|_{0} ^{L}+\left.\left[C_{2} w^{\prime \prime \prime}-Q(x)\right] \delta w^{\prime \prime}\right|_{0} ^{L}=0 .
\end{aligned}
$$

Equation (22) leads to the Euler-Lagrange equation of the variational formulation based on the total potential energy (16) for the Euler-Bernoulli beam equation derived under pure bending for MSGT as

$$
C_{1} \frac{d^{4} w}{d x^{4}}-C_{2} \frac{d^{6} w}{d x^{6}}-q(x)=0
$$

along with the relations between the force terms and displacement field at the boundaries

$$
V(x)=-C_{1} \frac{d^{3} w}{d x^{3}}+C_{2} \frac{d^{5} w}{d x^{5}}, \quad M(x)=C_{1} \frac{d^{2} w}{d x^{2}}-C_{2} \frac{d^{4} w}{d x^{4}}, \text { and } \quad Q(x)=C_{2} \frac{d^{3} w}{d x^{3}} .
$$

Equation (23) can be considered as the strong form of the static force balance which also enforces the moment balance (due to the symmetry of the stress tensor). The terms in equation (24) can be considered as the beam counterpart for the Cauchy stress theorem which relates the stresses and surface tractions in solids in MSGT.

While constructing the Boundary Conditions (BC's), the following should be defined:

- either a Dirichlet BC for vertical displacement $w$ or a Neumann BC for shear force $V$,

- either a Dirichlet $\mathrm{BC}$ for rotation $\theta$ or a Neumann $\mathrm{BC}$ for bendig moment $M$,

- either a Dirichlet BC for curvature $\kappa$ or a Neumann BC for higher order moment $Q$ (the nonclassical BC),

where $w, \theta$, and $\kappa=\frac{d^{2} w(x)}{d x^{2}}$ correspond to vertical displacement, rotation, and curvature, respectively, see also Figure 2.

(a)
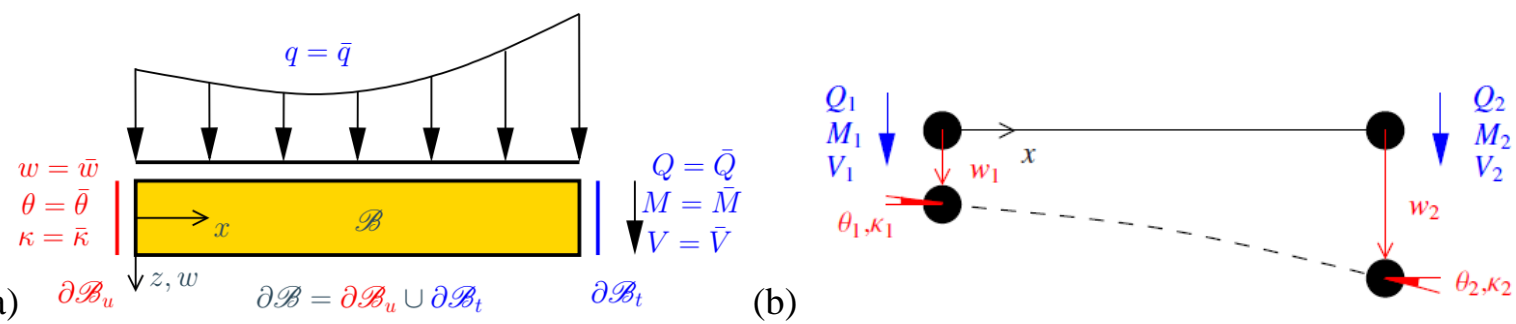

Figure 2. (a) The boundary $\partial \mathscr{B}=\partial \mathscr{B}_{u} \cup \partial \mathscr{B}_{t}$ is decomposed into Dirichlet (essential) $\partial \mathscr{B}_{u}$ and Neumann (natural) type conditions $\partial \mathscr{B}_{t}$ for a beam in MSGT. Dirichlet boundary conditions require the specification of the displacement, rotation and curvature $\{w, \theta, \kappa\}$ whereas, the Neumann boundary conditions require the specification of shear force, bending moment, and higher order moment $\{V, M, Q\}$, respectively. (b) Undeformed and deformed configurations of a beam element.

\subsection{Weak Formulation and Finite Element Discretization}

The analytical solution of equation (23) for an Euler-Bernoulli beam under certain boundary conditions is given by Kong et al. [26], and Akgöz and Civalek [31]. Relevant solutions can be derived for other boundary conditions and varying cross sections as well. However, given real life applications of micro- 
beams in MEMS and NEMS structures [1,2], finite element analysis is less tedious and easier to apply for complex boundary conditions involving forces and displacements, for both design and analysis purposes, and therefore is the focus of this research. In this section, the stiffness matrix of an EulerBernoulli beam based on MSGT is derived.

$$
\begin{gathered}
\delta \Pi=\frac{1}{2} \int_{0}^{L}\left[w^{\prime \prime} C_{1} \delta w^{\prime \prime}+w^{\prime \prime \prime} C_{2} \delta w^{\prime \prime \prime}\right] d x \\
-\int_{0}^{L} q(x) \delta w d x+\left.[V(x) \delta w]\right|_{0} ^{L}+\left.\left[M(x) \delta w^{\prime}\right]\right|_{0} ^{L}+\left.\left[Q(x) \delta w^{\prime \prime}\right]\right|_{0} ^{L}=0 .
\end{gathered}
$$

Herein, we define curvature $\kappa$ as an additional field variable for each node of the 2-node beam element (corresponding conjugate of which is $Q(x)$ in equation (23) in addition to vertical displacement $w$ and rotation $\theta$. Recall that by replacing $v(x)=\delta w$, one can easily show the equivalence of the weak form (25) and the first variation (20) of the total potential energy functional (16) by multiplying the beam equation (23) with a test function $v(x)=\delta w$ and integrating over the length of beam element $L$, and then using integration by parts and reorganizing terms.

In order to solve the equation (25), the finite element method will $b$ used. To do so, the nodal vector of generalized displacements is defined

$$
\boldsymbol{D}^{k}=\left[\begin{array}{llllll}
w_{1} & \theta_{1} & \kappa_{1} & w_{2} & \theta_{2} & \kappa_{2}
\end{array}\right]^{T}
$$

(a)

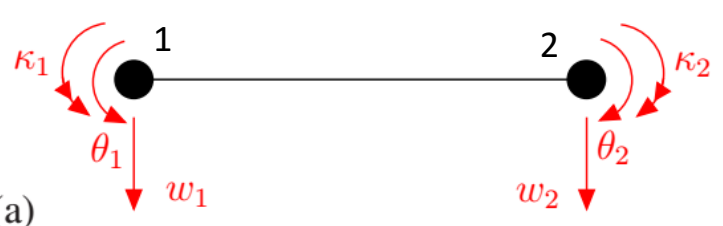

(b)

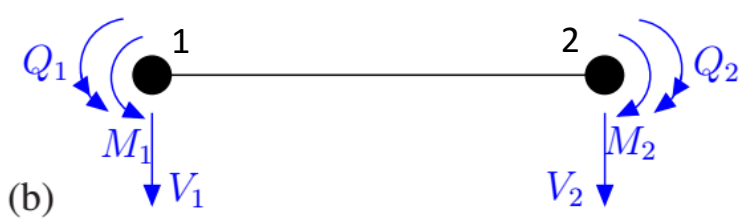

Figure 3. (a) Nodal degrees of freedom and (b) nodal forces acting on a higher order beam element

$$
\boldsymbol{N}(\boldsymbol{x})=\left[\begin{array}{llllll}
N_{1}(x) & N_{2}(x) & N_{3}(x) & N_{4}(x) & N_{5}(x) & N_{6}(x)
\end{array}\right]
$$

which interpolate the displacement field within the element. A special choice of shape functions

$$
w(x)=a_{1}+a_{2} x+a_{3} x^{2}+a_{4} x^{3}+a_{5} \cosh \left(x \sqrt{\frac{c_{1}}{c_{2}}}\right)+a_{6} \sinh \left(x \sqrt{\frac{c_{1}}{C_{2}}}\right)
$$

satisfy the solution of the partial differential equation (23) for $q(x)=0$. Moreover, the following relations for the vertical displacement, rotation, curvature fields

$$
w(x)=\boldsymbol{N}(x) \boldsymbol{D} \quad \theta(x):=w^{\prime}(x)=\boldsymbol{N}^{\prime}(x) \boldsymbol{D} \quad \kappa(x):=w^{\prime \prime}(x)=\boldsymbol{N}^{\prime \prime}(x) \boldsymbol{D},
$$

can be established along with their variations

$$
\delta w(x)=\boldsymbol{N}(x) \delta \boldsymbol{D} \quad \delta \theta(x):=\delta w^{\prime}(x)=\boldsymbol{N}^{\prime}(x) \delta \boldsymbol{D} \quad \delta \kappa(x):=\delta w^{\prime \prime}(x)=\boldsymbol{N}^{\prime \prime}(x) \delta \boldsymbol{D}
$$

or

$$
\delta w(x)=\delta \boldsymbol{D}^{T} \boldsymbol{N}(x)^{T} \quad \delta w^{\prime}(x)=\delta \boldsymbol{D}^{T} \boldsymbol{N}^{\prime}(x)^{T} \quad \delta w^{\prime \prime}(x)=\delta \boldsymbol{D}^{T} \boldsymbol{N}^{\prime \prime}(x)^{T}
$$


with $\boldsymbol{N}^{\prime}(x)=\frac{d \boldsymbol{N}(x)}{d x}$ and $\boldsymbol{N}^{\prime \prime}(x)=\frac{d^{2} \boldsymbol{N}(x)}{d x^{2}}$. The shape functions (27) which satisfy the displacement function (28) are derived with the help of the MATLAB by symbolic math toolbox and are given in Appendix. Incorporation of the interpolation functions within the element subdomain for displacement $w^{\prime \prime}(x), w^{\prime \prime \prime}(x)$ and their variations $\delta w^{\prime \prime}(x), \delta w^{\prime \prime \prime}(x)$ into (25) one obtains the discrete counterpart

$$
\bigcup_{k=1}^{n_{\text {elem }}}\left\{\delta \boldsymbol{D}^{k T}\left\{\int_{0}^{L_{e}}\left[C_{1}\left(\frac{d^{2} \boldsymbol{N}(x)}{d x^{2}}\right)^{T}\left(\frac{d^{2} \boldsymbol{N}(x)}{d x^{2}}\right)\right]+\left[\boldsymbol{C}_{2}\left(\frac{d^{3} \boldsymbol{N}(x)}{d x^{3}}\right)^{T}\left(\frac{d^{3} \boldsymbol{N}(x)}{d x^{3}}\right)\right] d x\right\} \boldsymbol{D}^{k}\right\}
$$

of the continuous functional (25). The variational discrete displacement vector $\delta \boldsymbol{D}^{k}$ vanishes at essential boundaries (the global vector has to have zero entries at essential boundaries) where $\boldsymbol{D}=\left.\overline{\boldsymbol{D}}\right|_{\partial \mathscr{G} u}$ is prescribed. The equality (32) must hold for arbitrary variation $\delta \boldsymbol{D}^{\mathrm{g}} \cup_{k=1}^{n_{\text {elem }}} \delta \boldsymbol{D}^{k}$. Therefore, for linear problems, the continuous system (25) reduces to a set of linear algebraic equations in the form

$$
\boldsymbol{K}^{\mathrm{g}} \cdot \boldsymbol{D}^{\mathrm{g}}=\boldsymbol{F}^{\mathrm{g}}
$$

where $\boldsymbol{K}^{\mathrm{g}}=\cup_{k=1}^{n_{\text {elem }}} \boldsymbol{K}^{k}$ is the global stiffness matrix, $\boldsymbol{D}^{\mathrm{g}}=\cup_{k=1}^{n_{\text {elem }}} \boldsymbol{D}^{k}$ is the global displacement vector, and $\boldsymbol{F}^{\mathrm{g}}=\bigcup_{k=1}^{n_{\text {elem }}} \boldsymbol{F}^{k}$, respectively. The element stiffness matrix $\boldsymbol{K}^{e}$ is evaluated as

$$
\boldsymbol{K}^{e}=\int_{0}^{L_{e}}\left[\left(\frac{d^{2} N(x)}{d x^{2}}\right)^{T} C_{1}\left(\frac{d^{2} N(x)}{d x^{2}}\right)+\left(\frac{d^{3} N(x)}{d x^{3}}\right)^{T} C_{2}\left(\frac{d^{3} N(x)}{d x^{3}}\right)\right] d x .
$$

Element nodal force vector can also be defined as

$$
\boldsymbol{F}^{\mathrm{e}}=\int_{0}^{L_{e}}\left[\boldsymbol{N}(x)^{T} q(x)\right] d x+\boldsymbol{F}^{\mathrm{d}},
$$

where $\boldsymbol{F}^{\mathrm{d}}=\left[\mathrm{V}_{1} \mathrm{M}_{1} \mathrm{Q}_{1} \mathrm{~V}_{2} \mathrm{M}_{2} \mathrm{Q}_{2}\right]$ is the element nodal force vector. The second $\boldsymbol{N}^{\prime \prime}(x)$ and third $\boldsymbol{N}^{\prime \prime \prime}(x)$ derivatives of the shape functions $\boldsymbol{N}(x)$ can be derived in a straightforward manner leading to lengthy expressions which are omitted from the manuscript. This has been carried out with the MATLAB symbolic toolbox. The elements of the row vector for shape functions $\boldsymbol{N}(x)$ are given in the Appendix. In the limiting case, where all the length scale parameters $l_{0}, l_{1}$, and $l_{2}$ vanish, MSGT formulation tends to the classical theory as leading to $C_{1} \rightarrow E^{\prime} I$ and $C_{2} \rightarrow 0$. Then, the well-known element stiffness matrix for classical Euler-Bernoulli beam theory is achieved as

$$
\boldsymbol{K}^{e}=\int_{0}^{L_{e}}\left[\left(\frac{d^{2} \widehat{N}(x)}{d x^{2}}\right)^{T} E I\left(\frac{d^{2} \widehat{N}(x)}{d x^{2}}\right)\right] d x
$$

The sahape functions $\widehat{\boldsymbol{N}}(x) \rightarrow\left[\begin{array}{llll}\widehat{N}_{1} & \widehat{N}_{2} & \widehat{N}_{3} & \widehat{N}_{4}\end{array}\right]$ reduce to the classical third order Hermite cubic polynomials given in the Appendix.

\section{NUMERICAL ANALYSIS AND PARAMETER IDENTIFICATION}

The finite element method outlined in Section 2 is implemented into MATLAB and used for the subsequent investigations carried out in this section using double cantilever and cantilever beams illustrated in Figure 4. The finite element codes developed in this study are verified using the values reported in Lam et al. [25], Kong et al. [26] and Kahrobaiyan et al. [28]. A number of experiments are conducted on gold micro- and nano-beams and reported in the literature [47]. The linear elastic portions of the load-displacement curves reported in the literature are used for the parameter identification of the elastic constants of the modified strain gradient theory for gold micro-beams. 
Several remarks on the selection of the experimental data and the parameter identification:

- There are actually various experiments published with gold specimens in the literature, however as they don't present raw load-displacement curves but stress-strain curves (based on classical theories), they are not incorporated in the study.

- The analysis will focus on the extraction of the length scale parameters $\left(l_{0}, l_{1}, l_{2}\right)$ for gold in order to obtain the best fit to the load-displacement curves for beams with various thicknesses.

- For the sake of convenience, the length scale parameters $\left(l_{0}, l_{1}, l_{2}\right)$ are taken identical for MSGT (as in many studies including the breakthrough study of Lam et al. [25]) and will be referred to as $l$.

- Among all the experimental data in the literature, the investigation the data provided by Espinosa et al. [47] is the only one which provides sufficient information for the extraction of the length-scale parameter for gold. Therein, load-displacement curves for double cantilever beam with two different thicknesses are presented. The dimensions of the specimen geometries are depicted in Figure 4 and Table 1.

- The finite element mesh for the double cantilever beam and the cantilever beam used throughout the simulations consists of 10 beam elements.
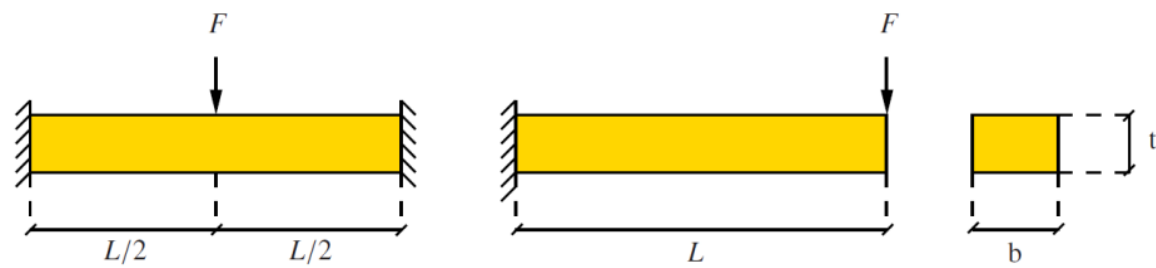

Figure 4. Illustration of double cantilever (left) and cantilever beams (middle) along with their cross section (right). $\mathrm{F}$ is a point load applied to the middle of the double cantilever beam, and free end of the cantilever beam

Table 1. Specimen dimensions and force-displacement values for the double cantilever beams reported in Espinosa et al. [47]. $b, t, L$ denote the width, thickness and length of the specimen respectively. $F$ and $w$ are the vertical force and the actual mid-point displacement, respectively. (*gage dimension)

\begin{tabular}{|c|c|c|c|c|c|c|}
\hline Type & Specimen \# & $\begin{array}{c}b \\
{[\mu \mathrm{m}]}\end{array}$ & $t[\mu \mathrm{m}]$ & $L[\mu \mathrm{m}]$ & $F[\mathrm{mN}]$ & $w[\mu \mathrm{m}]$ \\
\hline Double-cantilever & 1 & $10^{*}$ & 0.5 & $400^{*}$ & 0.3 & 15 \\
\hline Double-cantilever & 2 & $10^{*}$ & 1 & $400^{*}$ & 0.3 & 9 \\
\hline
\end{tabular}

For the quantification of the best fit, an error function

$$
\operatorname{Err}(w)=\left(w^{\prime}{ }_{1}-w_{1}\right)^{2}+\left(w^{\prime}{ }_{2}-w_{2}\right)^{2}
$$

is defined as the L2-norm of the residual vector. Here $w_{1}^{\prime}$ and $w^{\prime}{ }_{2}$ are the mid-point deflections predicted by MSGT and $w_{1}$ and $w_{2}$ are the actual deflections from experiments, for specimens 1 and 2 respectively. The error function is evaluated for different values of $E$ and $l$ ( $E$ varying from $20 \mathrm{GPa}$ to $140 \mathrm{GPa}$ with a resolution of $1 \mathrm{GPa}, l$ varying from $2 \mu \mathrm{m}$ to $8 \mu \mathrm{m}$ with a resolution of $0.05 \mu \mathrm{m}$ ), in order to find the minimum error, see Figure 5. 


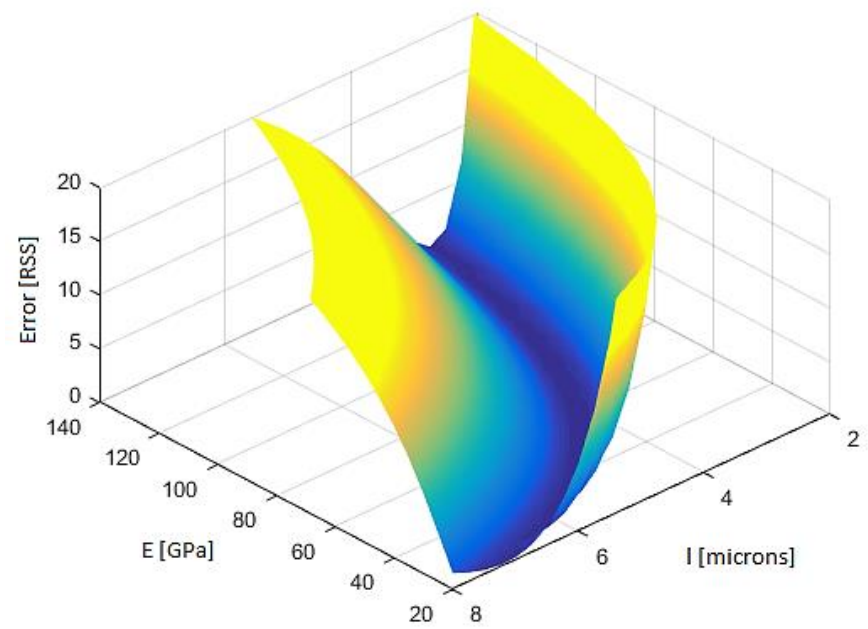

Figure 5. Error function (RSS) per various values of $E$ and $l$.

The minimum and maximum values of $E$ are chosen according to the upper and lower limits reported in the literature. It is seen that the error function is minimum along a curve as given in Figure 6 (corresponding to the dark blue region in Figure 5). It is also found that the error values are monotonically decreasing as $E$ decreases. Values smaller than E=20 GPa are excluded since they are not found realistic. Hence a realistic evaluation for $E$ and $l$ at the minimum error point could not be made. Instead, set of values for $E$ and $l$ are identified (which are in fact those corresponding to minimum error values given in Figure 5 and Figure 6). However, for all reported values, significant size effect is present $(l \in[2.70,7.15] \mu \mathrm{m})$.

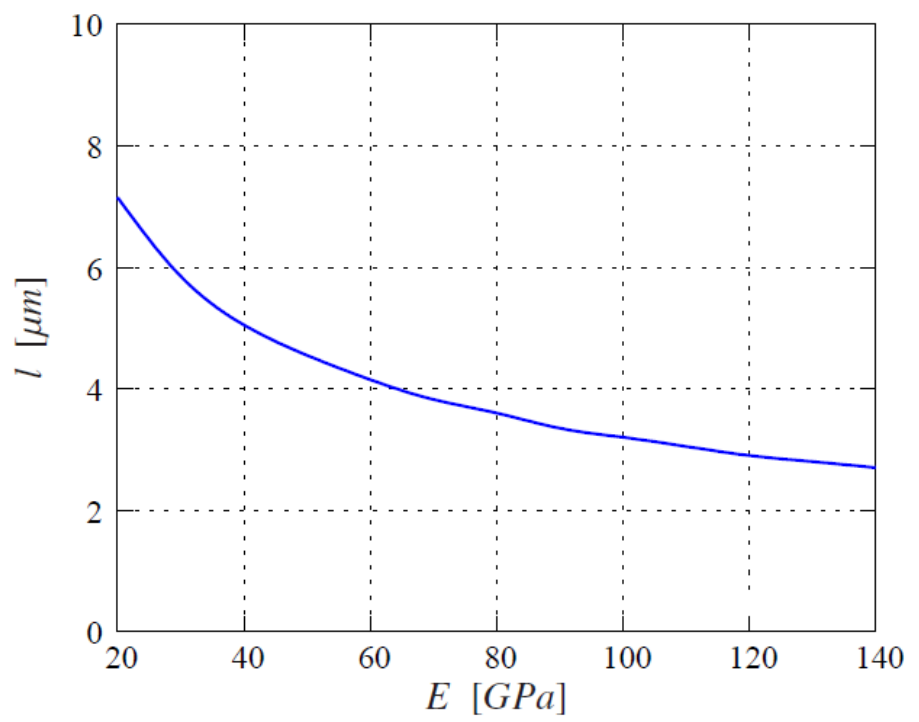

Figure 6. $E[\mathrm{MPa}]$ and $l[\mu \mathrm{m}]$ values corresponding to minimum values of error function

It is found that the minimum error for bulk elastic modulus of gold ( $E=80 \mathrm{GPa}), l$ is found as $3.60 \mu \mathrm{m}$, whereas for arbitrary values of $E=20 \mathrm{GPa}$ and $140 \mathrm{GPa}$, the length scale parameters are $7.15 \mu \mathrm{m}$ and $2.70 \mu \mathrm{m}$ respectively. It must also be noted that the error margin for these length scale parameters is $\pm 0.025 \mu \mathrm{m}$ because of the resolution of the length scale parameter in the analysis. For the sake of convenience, these length scale parameters are to be referred as $l_{20}$ (corresponding to $E=20 \mathrm{GPa}$ ), as $l_{80}$ (corresponding to $E=80 \mathrm{GPa}$ ), and $l_{140}$ (corresponding to $E=140 \mathrm{GPa}$ ). 
This size effect, which is characterized with length scale parameters is crucial in determining actual beam behavior. The ratio of maximum deflections for a double-cantilever beam (i.e. at the middle node) predicted by classical theory $\left(w_{0}{ }^{\prime}\right)$ and MSGT $\left(w^{\prime}\right)$ are drawn in Figure 7 below with various thicknesses, with width $b=5 t$, and length $L=4 b, F=30 \mathrm{mN}$ as found with the developed codes.

It is seen that the MSGT asymptotically approaches to the classical theory as the thickness, which is related to the structural length scale, increases. With the assumed thickness-width-length ratio (1:5:20) for gold micro-beams, the error of using classical theory with macroscopic material parameters in predicting beam deflections is more than $10 \%$ if thickness is reduced below $t=\{16,32\} \mu \mathrm{m}$ for $E=\{80,140\}$ $\underline{G P a}$. Further analysis shows that variation in the thickness-width-length ratio does not affect these values significantly and beam thickness is seen to be the critical parameter controlling the size effect.

There is also another distinctive feature of MSGT that separates it from classical beam theory. In classical theory, deflection under a constant force is directly proportional to $L^{3}$ and inversely proportional to $E I=E b t^{3} / 12$. When thickness-width-length proportionality is preserved, this results in a directly proportional decrease in deflections with increasing dimensions, as given in Figure 8. In MSGT, however, there is a particular size for the same thickness-width-length ratio for which there is a maximum deflection $(t=10 \mu \mathrm{m}$ in this example for $E=80 \mathrm{GPa})$.

Moreover, between zero and this maximum deflection point (for constant thickness-width-length ratio), the trends in deflections predicted by classical beam theory and MSGT are completely reversed (a decrease in classical theory, an increase in MSGT). This aspect, if explored further with experiments and if found compatible, can be made use of for finding optimum shapes and dimensions for various MEMS applications.

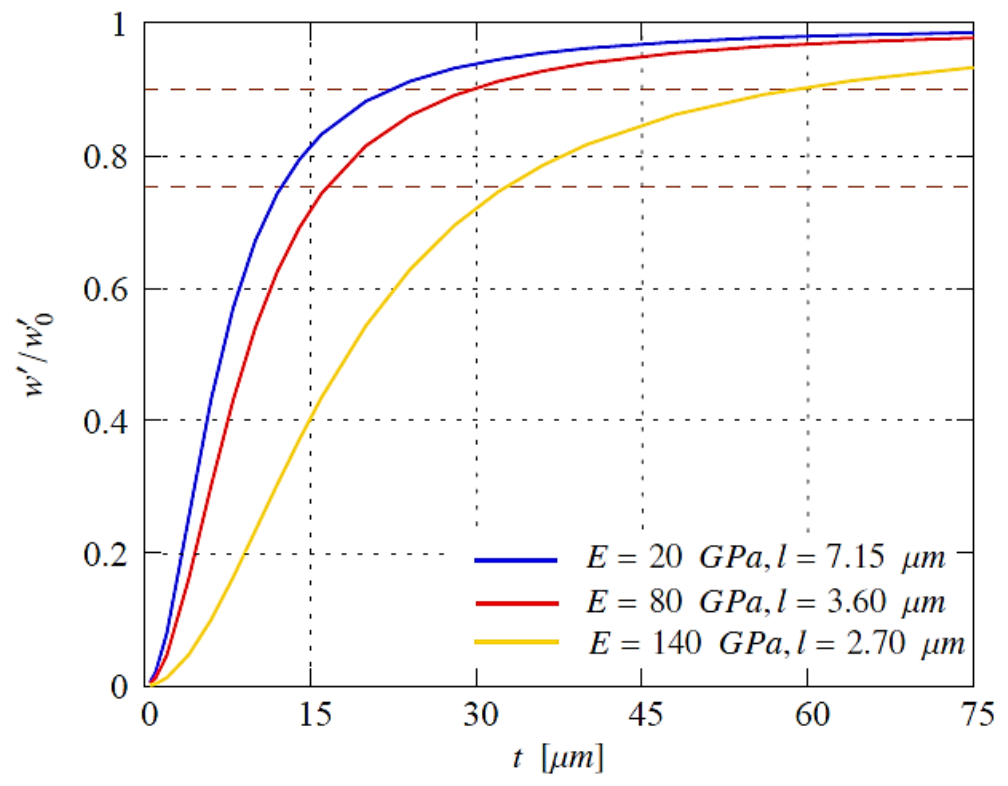

Figure 7. The ratio of mid-point deflection predicted by MSGT $\left(w^{\prime}\right)$ to that predicted by classical theory $\left(w_{0}{ }^{\prime}\right)$ with $l=3.60 \mu \mathrm{m}\left(l_{80}\right)$, for geometrical parameters and the force applied to the middle node as defined, and the corresponding thickness level for an error of $10 \%$ and $25 \%$ respectively. The figures for $l_{20}$ and $l_{140}$ are also shown with dashed lines.

In order to further emphasize the effects of the size effect, the following case, this time for a cantilever beam is also run, as given in Table 2 . 

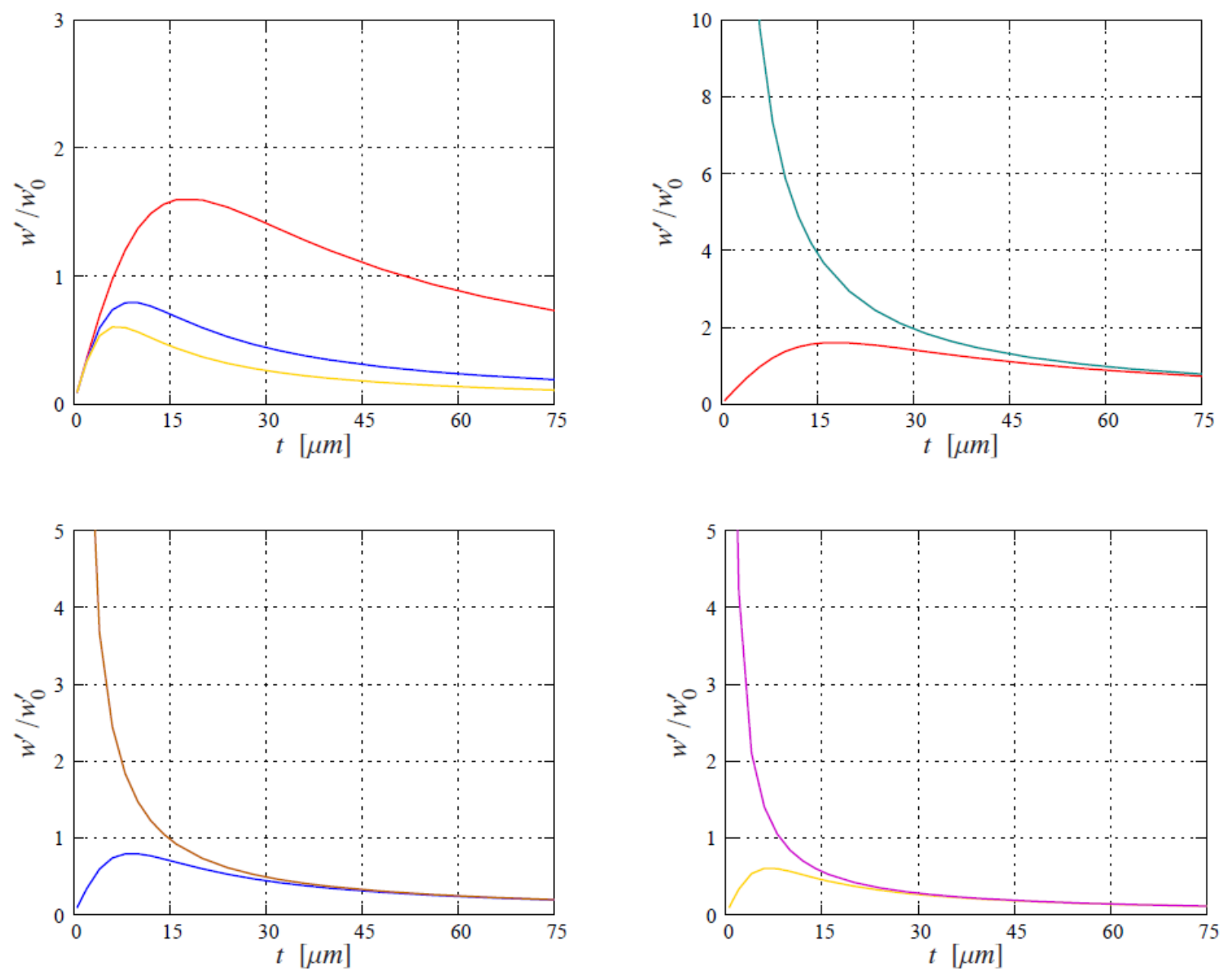

$$
\begin{aligned}
-M S G T, E=20 \mathrm{GPa}, l=7.15 \mu \mathrm{m} & \text { EBBT,E}=20 \mathrm{GPa} \\
M S G T, E=80 \mathrm{GPa}, l=3.60 \mu \mathrm{m} & -E B B T, E=80 \mathrm{GPa} \\
M S G T, E=140 \mathrm{GPa}, l=2.70 \mu \mathrm{m} & \text { EBBT, E }=140 \mathrm{GPa}
\end{aligned}
$$

Figure 8. The deflections predicted by MSGT $\left(w^{\prime}\right)$ (for $l_{20}, l_{80}$, and $\left.l_{140}\right)$, and by classical Euler Bernoulli Beam Theory $\left(w_{0}{ }^{\prime}\right)$ for geometrical parameters and the force applied to the middle node as defined above.

In Figure 9, the deflected shapes for the beam with the geometric properties given in Table 2, , are drawn according to classical theory and MSGT. The results are normalized with respect to the maximum deflection at the free node for classical theory, length of the beam normalized by diving to the overall beam length. The examples can be varied to show the size effect in beam bending; the outcome of would be the principle of increasing beam stiffness as beam sizes decrease.

Table 2. Selected beam to show size effect under a vertical load of $1 \mathrm{mN}$ applied on the free node of the cantilever beam

\begin{tabular}{|c|c|c|c|}
\hline type & $b[\mu \mathrm{m}]$ & $t[\mu \mathrm{m}]$ & $L[\mu \mathrm{m}]$ \\
\hline Cantilever & 20 & 4 & 80 \\
\hline
\end{tabular}




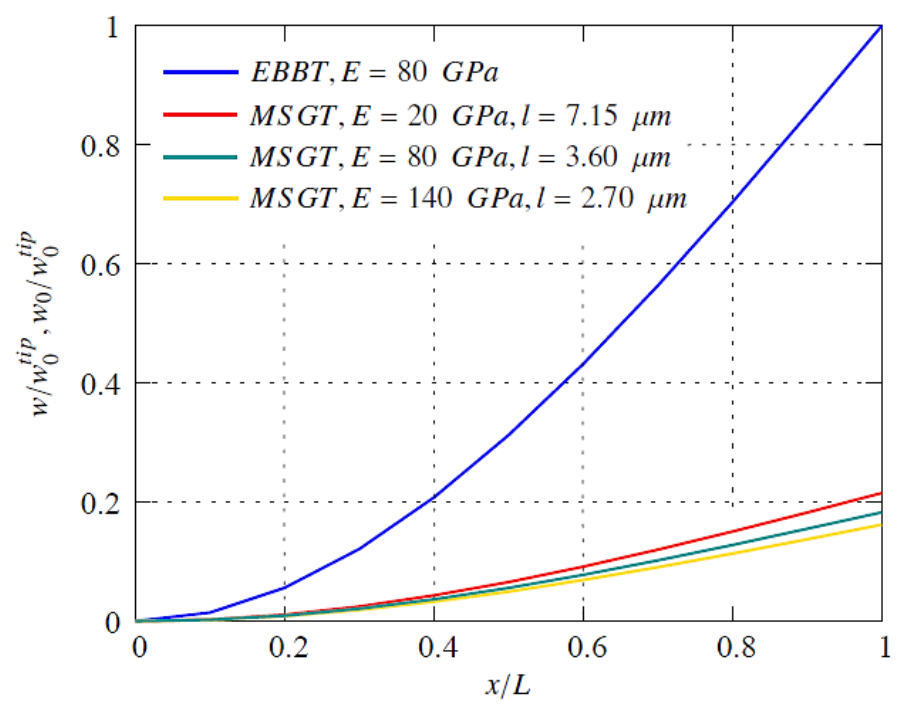

Figure 9. The deflections predicted by classical theory (EBBT) (denoted as $w_{0}$ ) and MSGT (denoted as $w$ ) divided by tip deflection predicted by classical theory $w_{0}^{\text {tip }}$, for the specimen with geometric properties given in Table 2.

The length scale parameters for three different assumptions for $E^{\prime}$ are outlined in Table 3, where $E=80$ GPa.

Table 3. Length scale parameters identified with different assumptionf for the parameter $E^{\prime}$.

\begin{tabular}{|l|c|}
\hline Formulation & $\boldsymbol{l}[\boldsymbol{\mu m}]$ \\
\hline$E^{\prime}=\frac{E(1-v)}{(1-2 v)(1+v)}$, Akgöz and Civalek [31] & 3.577 \\
\hline$E^{\prime}=E$, Kahrobaiyan et al. [28] & 3.582 \\
\hline$E^{\prime}=\frac{E}{\left(1-v^{2}\right)}$, Zhao et al. [35] & 3.581 \\
\hline
\end{tabular}

It must be noted that the plane stress or plane strain assumption do not affect the length scale parameter identified significantly. This is due to the fact that, the contribution of length scale parameters to the bending stiffness of the beam is considerably higher than that of the Young's modulus.

\section{REMARKS AND CONCLUSION}

In this contribution, a compact formulation of Euler-Bernoulli beam theory is proposed for the modified strain gradient theory of elasticity of Lam et al. [25]. The finite element formulation based on Galerkin approach is briefly introduced. As a novel aspect, the length scale parameter of gold is identified with the help of the experimental data reported by Espinosa et al. [47].

The comparison of the results of classical beam theory with MSGT clearly demonstrates the insufficiency of the classical beam theory at micron-scale. It is shown that for a double cantilever gold beam, the deviation in classical theory with bulk properties of gold is approximately $10 \%$ for a beam thickness of around $60 / 30 \mu \mathrm{m}$, and $25 \%$ for a beam thickness of around $32 / 16 \mu \mathrm{m}$ for elasticity modulus 
$\mathrm{E}=140 / 80 \mathrm{GPa}$. It can be concluded that higher-order theories should definitely be employed for beams with thickness smaller than $50 \mu \mathrm{m}$. From MSGT, the length scale parameter is identified to be in the range $l \in[2.7,7.15] \mu \mathrm{m}$. Unfortunately, due to lack of adequate number of experimental data performed on varying beam thickness, a unique set of parameters could not be obtained. For this purpose, the length scale parameter according to the selected modulus of elasticity parameters are identified by minimizing the error function described as L2-norm of the mid-point displacement of the double cantilever beam. However, all identified parameters demonstrate significant size effect.

It is crucial to mention that more experiments need to be undertaken with various dimensions as controlled parameters in order to verify length scale parameters. In this study, only two specimens with variable thicknesses $(0.5 \mu \mathrm{m}$ and $1 \mu \mathrm{m})$ are used, and more data is needed for different thicknesses. It may as well be the case that width and length of the beams should be taken as controlled parameters to ultimately verify that thickness is the sole parameter to be used along with bending parameter in order to come up with length scale parameters.

The length scale parameters for MSGT are taken as equal in this work as in many studies for simplification purposes $\left(l_{0}=l_{1}=l_{2}\right)$. However, more experimental data is required for reaching better fit between theory and experiment. This aspect can be elaborated in the future with the help of additional experimental data. This work clearly demonstrates the lack of sufficient experimental data on gold micro-beams at varying sizes and the necessity of the incorporation of higher-order beam theories for the analysis and design of NEMS-MEMS devices.

\section{ACKNOWLEDGEMENT}

The author would like to thank Murat Kandaz, M.Sc. for the stimulating discussions during this research. The financial support from TUBITAK under TUBİTAK 1001 scheme with grant number 116M258 is gratefully acknowledged.

\section{APPENDIX}

The shape functions mentioned in equation (27) and form the major part of the stiffness matrix in equation (31) are given as:

$$
\begin{aligned}
& N_{1}(s)=\left[12 \cosh (q(s-1))-12 \cosh (q s)+12 \cosh (q)+6 q^{2} s^{2}-4 q^{2} s^{3}+8 q^{2} \cosh (q)-q^{3} \sinh (q)+\right. \\
& 6 q \sinh (q s)-6 q 2 s-18 q \sinh (q)+4 q^{2}+6 q \sinh (q(s-1))+12 q \sinh (q)-6 q 2 \cosh (q)-6 q^{2} s^{2} \cosh (q) \\
& \left.+4 q^{2} s^{3} \cosh (q)+3^{q} 3 s^{2} \sinh (q)-2 q^{3} s^{3} \sinh (q)-12\right] / D \\
& N_{2}(s)=-\left[12 \sinh (q(s-1))-12 \sinh (q s)+12 \sinh (q)-2 q^{3} s^{3}+4 q^{2} \sinh (q)-12 q s+4 q^{2} \sinh (q(s-1))\right. \\
& +2 q^{3} s-12 q \cosh (q)+12 q \cosh (q(s-1))+2 q^{2} \sinh (q s)+12 q \operatorname{scosh}(q)+4 q^{3} \operatorname{scosh}(q)-12 q^{2} \sinh (q)- \\
& \left.q^{4} \sinh (q)-6 q^{3} s^{2} \cosh (q)+2 q^{3} s^{3} \cosh (q)+6 q^{2} s^{2} \sinh (q)+2 q^{4} s^{2} \sinh (q)-q^{4} s^{3} \sinh (q)\right] L /(-q D) \\
& N_{3}(s)=-\left[6 \sinh (q(s-1))-2 q-6 \sinh (q s)+6 \sinh (q)+q^{3} s^{2}-q^{3} s^{3}+q^{2} \sinh (q)+6 q s+q^{2} \sinh (q(s-\right. \\
& 1))+2 q \cosh \left(q \sin -12 q s^{2}+4 q s^{3}-4 q \cosh (q)+4 q \operatorname{coshh}(q(s-1))-6 q \operatorname{scosh}(q)+12 q s^{2} \cosh (q)-\right. \\
& \left.4 q s^{3} \cosh (q)-q^{3} \operatorname{scosh}(q)+4 q^{2} \sinh (q)+2 q^{3} s^{2} \cosh (q)-q^{3} s^{3} \cosh (q)-9 q^{2} s^{2} \sinh (q)+4 q^{2} s^{3} \sinh (q)\right] L^{2} \\
& /(-q D)
\end{aligned}
$$


$N_{4}(s)=-\left[12 \cosh (q(s-1))-12 \cosh (q s)-12 \cosh (q)+6 q^{2} s^{2}-4 q^{2} s^{3}+6 q \sinh (q s)-6 q^{2} s-6 q \sinh (q)\right.$ $+6 q \sinh (q(s-1))+12 q \operatorname{ssinh}(q)-6 q^{2} \operatorname{scosh}(q)-6 q^{2} s^{2} \cosh (q)+4 q^{2} s^{3} \cosh (q)+3 q^{3} s^{2} \sinh (q)-2 q^{3} s^{3}$ $\sinh (q)+12] / D$

$N_{5}(s)=-\left[12 q-12 \sinh (q(s-1))+12 \sinh (q s)-12 \sinh (q)+6 q^{3} s^{2}-2 q^{3} s^{3}+2 q^{2} \sinh (q)-12 q s+\right.$ $2 q^{2} \sinh (q(s-1))-12 q \cosh (q s)-4 q^{3} s+4 q^{2} \sinh (q s)+12 q \operatorname{scosh}(q)-2 q^{3} \operatorname{scosh}(q)+2 q^{3} s^{3} \cosh (q)-$ $\left.6 q^{2} s^{2} \sinh (q)+q^{4} s^{2} \sinh (q)-q^{4} s^{3} \sinh (q)\right] L /(-q D)$

$N_{6}(s)=-\left[6 \sinh (q(s-1))-4 q-6 \sinh (q s)+6 \sinh (q)-2 q^{3} s^{2}+q^{3} s^{3}+6 q s+4 q \cosh (q s)+4 q s^{3}+q^{3} s\right.$ $-2 q \cosh (q)+2 q \cosh (q(s-1))-q^{2} \sinh (q s)-6 q \operatorname{scosh}(q)+4 q s^{3} \cosh (q)+2 q^{2} \sinh (q)-q^{3} s^{2} \cosh (q)$ $\left.+q^{3} s^{3} \cosh (q)+3 q^{2} s^{2} \sinh (q)+4 q^{2} s^{3} \sinh (q)\right] L^{2} /(-q D)$

where:

$q=L / \sqrt{c_{1} / c_{2}}, s=x / L, D=24 \cosh (q)+8 q^{2} \cosh (q)-q^{3} \sinh (q)-24 q \sinh (q)+4 q^{2}-24$

In the limiting case where all the length scale parameters $l_{0}, l_{1}$, and $l_{2}$ vanish, MSGT formulation tends to the classical theory as leading to $C_{1} \rightarrow E^{\prime} I$ and $C_{2} \rightarrow 0$.

For vanishing length scale parameter $l$ The classical shape functions in the stiffness matrix in Equation (32) reduces to

$N_{1} \rightarrow \widehat{N}_{1}=1-3 s^{2}+2 s,{ }^{3} \quad N_{2} \rightarrow \widehat{N}_{2}=L s(1-s)^{2}, \quad N_{4} \rightarrow \widehat{N}_{3}=3 s^{2}(1-2 s), \quad N_{5} \rightarrow \widehat{N}_{4}=L s^{2}(s-1)$

$N_{3} \rightarrow 0, \quad N_{6} \rightarrow 0$.

\section{REFERENCES}

[1] Rebeiz GM, RF MEMS: Theory, Design and Technology, John Wiley \& Sons, Inc. New York, NY, 2003

[2] Unlu M, Hashemi M, Berry C, Li S, Yang S, Jarrahi M. Switchable scattering meta-surfaces for broadband terahertz modulation. Nature Scientific Reports 2014; 4: 5708

[3] Berry C, Wang N, Hashemi M, Unlu M and Jarrahi M. Significant performance enhancement in photoconductive terahertz optoelectronics by incorporating plasmonic contact electrodes. Nature Communications 2013; 4: 1622

[4] Maluf N, Williams K. Introduction to microelectromechanical systems engineering (second edition), Artech House Inc. 2004

[5] Stelmashenko NA, Walls MG, Brown LM, Millman YV. Microindentations on W and Mo oriented single crystals: an STM study. Acta Metallurgica et Materialia 1993; 41: 2855

[6] Fleck NA, Hutchinson JW. Phenomenological theory for strain gradient effects in plasticity. Journal of the Mechanics and Physics of Solids 1993; 41 (12): 1825-1857

[7] Ma Q, Clarke DR. Size Dependence of the Hardness of Silver Single Crystals. Journal of Materials Research 1995; 10: 853 
[8] Chasiotis I, Knauss WG. A new microtensile tester for the study of MEMS materials with the aid of atomic force microscopy. Experimental Mechanics. 2002; 42 (1): 51-57

[9] Weihs TP, Hong S, Bravman JC, Nix WD. Mechanical deflection of cantilever microbeams: A new technique for testing the mechanical properties of thin films. Journal of Materials Research 1988; 3: 931-942

[10] Greer JR, Nix WD. Size dependence of mechanical properties of gold at the sub-micron scale. Applied Physics A/Materials Science \& Processing 2005; 80 (8): 1625-1629

[11] Voigt W. Theoretische studien uber die elasticitätsverhaltnisse der krystalle. i. ableitung der grundgleichungen aus der annahme mit polarität begabter moleküle. Abhandlungen der Matematischen Classe der Königlichen Gesellshaft der Wissenschaften zu Göttingen 1887; 24: 3-52

[12] Mindlin RD, Tiersten HF. Effects of couple-stresses in linear elasticity. Archive for Rational Mechanics and Analysis 1962; 11: 415-448

[13] Grioli G. Elasticità asimmetrica. Annali di Matematic Pura ed Applicate 1960; 4-50: 389-417

[14] Aero E, Kuvshinskii E. Fudamental equations of the theory of elastic media with rotationally interacting particles. Fizika Tverdogo Tela 1960; 2: 1399-1409

[15] Eringen A. Theory of micropolar elasticity. Fracture 1968; 1: 621-729

[16] Nowacki, W. Theory of Micropolar Elasticity. Springer Science and Business Media 1970

[17] Toupin R. Elastic materials with couple stress. Archive for Rational Mechanics and Analysis 1962; 11: $385-414$

[18] Mindlin R. Micro-structure in linear elasticity. Archive for Rational Mechanics and Analysis 1964; 16

[19] Mindlin R. Second gradient of strain and surface-tension in linear elasticity. International Journal of Solids and Structures 1968; 1: 417-438

[20] Mindlin R, Eshel N. On first strain-gradient theories in linear elasticity. International Journal of Solids and Structures 1968; 4: 109-124

[21] Koiter WT. Couple stresses in the theory of elasticity. I and II. Proceedings of the Koninklijke Nederlandse Akademie Van Wetenschappen. Series B. 1964; 67: 17-44

[22] Fleck NA, Hutchinson JW. Strain gradient plasticity. Advances in Applied Mechanics 1997; 33: 296-358

[23] Fleck NA, Hutchinson JW. A reformulation of strain gradient plasticity. Journal of the Mechanics and Physics of Solids 2001; 49: 2245-2271

[24] Yang F, Chong ACM, Lam DCC, Tong, P. Couple stress based strain gradient theory for elasticity. International Journal of Solids and Structures 2002; 39: 2731-2743

[25] Lam DCC., Yang F, Chong ACM, Wang J, Tong P. Experiments and theory in strain gradient elasticity. Journal of the Mechanics and Physics of Solids 2003; 51: 1477-1508

[26] Kong S, Zhou S, Nie Z, Wang K. Static and dynamic analysis of micro beams based on strain gradient elasticity theory. International Journal of Engineering Science 2003; 47: 487-498 
[27] Wang B, Zhao J, Zhou S. A micro scale Timoshenko beam model based on strain gradient elasticity theory. European Journal of Mechanics-A/Solids 2010; 29: 591-599

[28] Kahrobaiyan MH, Asghari M, Ahmadian MT. Strain gradient beam element. Strain gradient beam element. Finite Elements in Analysis and Design 2013; 68: 63-75

[29] Kahrobaiyan MH, Asghari M, Rahaeifard M, Ahmadian MT. A non-linear strain gradient beam formulation. International Journal of Engineering Science 2011; 49: 1256-1267

[30] Akgöz B, Civalek Ö. Strain gradient elasticity and modified couple stress models for buckling analysis of axially loaded micro-scaled beams. International Journal of Engineering Science 2011; 49: $1268-1280$

[31] Akgöz B, Civalek Ö. Analysis of micro-sized beams for various boundary conditions based on the strain gradient elasticity theory. Archive of Applied Mechanics 2012; 82:423-443

[32] Akgöz B, Civalek Ö. A size-dependent shear deformation beam model based on the strain gradient elasticity theory. International Journal of Engineering Science 2013; 70: 1-14

[33] Akgöz B, Civalek Ö. A microstructure-dependent sinusoidal plate model based on the strain gradient elasticity theory. Acta Mechanica 2015; 226: 2277-2294

[34] Akgöz B, Civalek Ö. A novel microstructure-dependent shear deformable beam model. International Journal of Mechanical Sciences 2015; 99: 10-20

[35] Zhao J, Zhou S, Wang B, Wang X. Nonlinear microbeam model based on strain gradient theory. Applied Mathematical Modelling 2012; 36: 2674-2686

[36] Ashgari M, Kahrobaiyan MH, Nikfar M, Ahmadian MT. A size-dependent nonlinear Timoshenko microbeam model based on the strain gradient theory. Acta Mechanica 2012; 223: 1233-1249

[37] Vatankhah R, Kahrobaiyan MH, Alasty A, Ahmadian M. Nonlinear forced vibration of strain gradient microbeams, Applied Mathematical Modelling 2012; 37: 8363-8382

[38] Zhang B, He Y, Liu D, Gan Z, Shen L. Non-classical Timoshenko beam element based on the strain gradient elasticity theory. Finite Elements in Analysis and Design 2014; 79: 22-39

[39] Rahaeifard M, Ahmadian M, Firoozbakhsh K. A strain gradient based yield criterion. International Journal of Engineering Science, 2014; 77: 45-54

[40] Taati E, Najafabadi MM, Reddy JN. Size-dependent generalized thermoelasticity model for Timoshenko micro-beams based on strain gradient and non-Fourier heat conduction theories. Composite Structures 2014; 116: 595-611

[41] Triantafyllou A., Giannakopoulos A. Structural analysis using a dipolar elastic Timoshenko beam. European Journal of Mechanics - A/Solids 2013; 39: 218-228

[42] Ghayesh MH, Amabili M, Farokhi H. Nonlinear forced vibrations of a microbeam based on the strain gradient elasticity theory. International Journal of Engineering Science 2013; 63: 52-60

[43] Ansari R, Gholami S, Sahmani S. Free vibration analysis of size-dependent functionally graded microbeams based on the strain gradient Timoshenko beam theory. Composite Structures 2011; 94: $221-228$ 
[44] Liebold C, Müller WH. Comparison of gradient elasticity models for the bending of micromaterials. Computational Materials Science 2016; 116: 52-61

[45] Papargyri-Beskou, S, Tsepoura K, Polyzos D, Beskos D. Bending and stability analysis of gradient elastic beams. International Journal of Solid of Structures 2003; 40: 385-400

[46] Shojaeian M, Tadi Beni Y, Ataei H. Electromechanical buckling of functionally graded electrostatic nanobridges using strain gradient theory. Acta Astronautica 2016; 118: 62-71

[47] Espinosa HD, Prorok BC, Fischer M. A methodology for determining mechanical properties of freestanding thin films and MEMS materials. Journal of the Mechanics and Physics of Solids 2003; 51: $47-67$

[48] Mindlin RD. Second gradient of strain and surface tension in linear elasticity. International Journal of Solids and Structures 1965; 1: 417-438

[49] Ma HM, Gao XL, Reddy JN. A microstructure-dependent Timoshenko beam model based on a modified couple stress theory. Journal of the Mechanics and Physics of Solids 2008; 56: 3379-3391

[50] Dehrouyeh-Semnani AM, Nikkhah-Bahrami, MA discussion on incorporating the Poisson effect in microbeam models based on modified couple stress theory. International Journal of Engineering Science 2015; 86: 20-25 